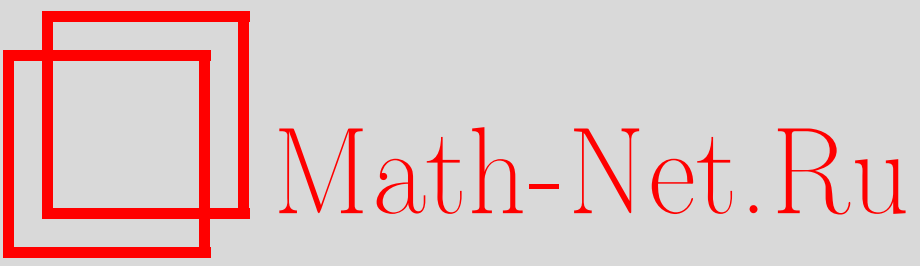

А. Я. Мальцев, Усреднение гамильтоновых структур в дискретном варианте метода Уизема, УМН, 1998, том 53, выпуск 1, 219-220

DOI: https://doi.org/10.4213/rm25

Использование Общероссийского математического портала Math-Net.Ru подразумевает, что вы прочитали и согласны с пользовательским соглашением

http://www. mathnet.ru/rus/agreement

Параметры загрузки:

IP : 54.198 .187 .58

26 апреля 2023 г., 15:07:07 


\title{
УСРЕДНЕНИЕ ГАМИЛЬТОНОВЫХ СТРУКТУР \\ В ДИСКРЕТНОМ ВАРИАНТЕ МЕТОДА УИЗЕМА
}

\author{
А. Я. МАЛЬЦЕВ
}

Данная работа посвящена построению процедуры усреднения гамилтоновых структур в дискретном варианте метода Уизема, являющейся аналогом процедуры Дубровина-Новикова для усреднения локальных теоретико-полевых скобок. Дискретным вариантом метода Уизема $([1],[2])$ мы будем назьвать получение уравнений эволюции медленно промодулированных $m$-фазных решений нелинейных систем

$$
\dot{\varphi}_{n}^{i}=f^{i}\left(\boldsymbol{\varphi}_{n}, \boldsymbol{\varphi}_{n-1}, \boldsymbol{\varphi}_{n+1}, \ldots\right),
$$

определенных на пространстве полей $\varphi=\left(\varphi_{n}^{1}, \ldots, \varphi_{n}^{p}\right)$, где $n$ принимает все целочисленные значения, функции $f$ зависят от конечного числа аргументов.

Квазипериодическими $m$-фазными решениями системы (1) называются решения вида

$$
\varphi_{n}^{i}(t)=\Phi^{i}\left(\mathbf{k}(\mathbf{U}) n+\boldsymbol{\omega}(\mathbf{U}) t+\theta_{0}, \mathbf{U}\right),
$$

где $\mathbf{k}(\mathbf{U})$ и $\boldsymbol{\omega}(\mathbf{U})-m$-мерные векторы, $\mathbf{U}=\left(U^{1}, \ldots, U^{N}\right)$ - параметры решения, а $2 \pi$-периодические функции $m$ переменных $(\theta) \Phi^{i}(\theta, \mathbf{U})$ удовлетворяют условиям:

$$
\omega^{\alpha}(\mathbf{U}) \Phi_{\theta^{\alpha}}^{i}(\theta, \mathbf{U})=f^{i}(\mathbf{\Phi}(\theta, \mathbf{U}), \boldsymbol{\Phi}(\theta-\mathbf{k}(\mathbf{U}), \mathbf{U}), \boldsymbol{\Phi}(\theta+\mathbf{k}(\mathbf{U}), \mathbf{U}), \ldots) .
$$

Пусть система (1) является гамильтоновой со скобкой Пуассона

$$
\left\{\varphi_{n}^{i}, \varphi_{k}^{j}\right\}=\sum_{m} B_{(m)}^{i j}\left(\varphi_{n}, \varphi_{n-1}, \varphi_{n+1}, \ldots\right) \delta_{n, k-m}
$$

где сумма содержит конечное число слагаемых (описание таких скобок см. в [3]), с гамильтонианом:

$$
H[\varphi]=\sum_{m=-\infty}^{\infty} \mathscr{P}_{H}\left(\boldsymbol{\varphi}_{n}, \boldsymbol{\varphi}_{n-1}, \boldsymbol{\varphi}_{n+1}, \ldots\right),
$$

и имеет $N$ инволютивных первых интегралов вида:

$$
I^{\nu}=\sum_{n=-\infty}^{\infty} \mathscr{P}^{\nu}\left(\boldsymbol{\varphi}_{n}, \boldsymbol{\varphi}_{n-1}, \boldsymbol{\varphi}_{n+1}, \ldots\right), \quad \nu=1, \ldots, N
$$

$$
\left\{I^{\nu}, I^{\mu}\right\}=0, \quad\left\{I^{\nu}, H\right\}=0
$$

$\left(B_{(m)}^{i j}, \mathscr{P}_{H}\right.$ и $\mathscr{P}^{\nu}$ зависят от конечного числа аргументов).

В силу (1) при этом будет:

$$
\frac{d}{d t} \mathscr{P}^{\nu}\left(\boldsymbol{\varphi}_{n}, \boldsymbol{\varphi}_{n-1}, \ldots\right)=\sum_{m}\left[Q_{(m)}^{\nu}\left(\boldsymbol{\varphi}_{n}, \boldsymbol{\varphi}_{n-1}, \ldots\right)-Q_{(m)}^{\nu}\left(\boldsymbol{\varphi}_{n-m}, \boldsymbol{\varphi}_{n-m-1}, \ldots\right)\right]
$$

(сумма содержит конечное число слагаемых, $Q_{(m)}^{\nu}$ зависят от конечного числа аргументов). Систему Уизема, описьвающую эволюцию медленно промодулированных параметров $\mathbf{U}$, можно записьвать в виде

$$
\frac{\partial}{\partial T}\left\langle\mathscr{P}^{\nu}\right\rangle(\mathbf{U}(X))=\sum_{m} m \frac{\partial}{\partial X}\left\langle Q_{(m)}^{\nu}\right\rangle(\mathbf{U}(X)),
$$

где $\langle\cdot\rangle$ означает усреднение на семействе $(2)$, определяемое формулой:

$$
\left\langle F\left(\boldsymbol{\varphi}_{n}, \boldsymbol{\varphi}_{n-1}, \ldots\right)\right\rangle(\mathbf{U})=\left(\frac{1}{2 \pi}\right)^{m} \int F(\boldsymbol{\Phi}(\theta, \mathbf{U}), \boldsymbol{\Phi}(\theta-\mathbf{k}(\mathbf{U}), \mathbf{U}), \ldots) d^{m} \theta
$$

Работа выполнена при финансовой поддержке Российского фонда фундаментальных исследований (грант № 96-01-01623). 
В силу (4) будем иметь:

$$
\left\{\mathscr{P}^{\nu}\left(\boldsymbol{\varphi}_{n}, \ldots\right), \mathscr{P}^{\mu}\left(\boldsymbol{\varphi}_{k}, \ldots\right)\right\}=\sum_{m} A_{(m)}^{\nu \mu}\left(\boldsymbol{\varphi}_{n}, \boldsymbol{\varphi}_{n-1}, \ldots\right) \delta_{n, k-m}
$$

причем, в силу (7),

$$
\sum_{m} A_{(m)}^{\nu \mu}\left(\boldsymbol{\varphi}_{n}, \boldsymbol{\varphi}_{n-1}, \ldots\right)=\sum_{l}\left(Q_{(l)}^{\nu \mu}\left(\boldsymbol{\varphi}_{n}, \boldsymbol{\varphi}_{n-1}, \ldots\right)-Q_{(l)}^{\nu \mu}\left(\boldsymbol{\varphi}_{n-l}, \boldsymbol{\varphi}_{n-l-1}, \ldots\right)\right)
$$

(все суммы содержат конечное число слагаемых, $A_{(m)}^{\nu \mu}$ и $Q_{(l)}^{\nu \mu}$ зависят от конечного числа аргументов).

Теорема 1. Пусть (без нарушения общности) параметры U выбраны так, что $U^{\nu}=\left\langle\mathscr{P}^{\nu}\right\rangle$. Тогда:

1) система (8) является гамильтоновой относительно скобки Пуассона гидродинамического типа (см. [4]-[5]), задаваемой соотношениями

$$
\left\{U^{\nu}(X), U^{\mu}(Y)\right\}=\sum_{m} m\left\langle A_{(m)}^{\nu \mu}\right\rangle(X) \delta^{\prime}(X-Y)+\left[\frac{\partial}{\partial X} \sum_{l} l\left\langle Q_{(l)}^{\nu \mu}\right\rangle(X)\right] \delta(X-Y)
$$

с гамильтонианом $H=\int\left\langle\mathscr{P}_{H}\right\rangle(X) d X$;

2) в силу (10) выполняются соотношения

$$
\begin{gathered}
\left\{k^{\alpha}(\mathbf{U}(X)), U^{\nu}(Y)\right\}=\omega^{\alpha(\nu)}(\mathbf{U}(X)) \delta^{\prime}(X-Y)+\frac{\partial \omega^{\alpha(\nu)}(\mathbf{U}(X))}{\partial X} \delta(X-Y), \\
\left\{k^{\alpha}(\mathbf{U}(X)), k^{\beta}(\mathbf{U}(Y))\right\}=0, \quad \alpha, \beta=1, \ldots, m, \quad \nu=1, \ldots, N,
\end{gathered}
$$

где $\boldsymbol{\omega}^{(\nu)}(\mathbf{U})$ - частоты, аналогичные $\boldsymbol{\omega}$, соответствующие потокам, порождаемым интегралами $I^{\nu}$ на семействе решений (2).

ТЕОрема 2. Пусть у системь (1) имеется два несовпадающих набора интегралов вида (6), $\left\{I^{1}, \ldots, I^{N}\right\}$ и $\left\{\bar{I}^{1}, \ldots, \bar{I}^{N}\right\}$, обладающих всеми требуемыми свойствами. Тогда скобки (10), полученные с помощью этих наборов, совпадают, т.е. если на семействе (2) $U^{\nu}=I^{\nu}, \bar{U}^{\nu}=\bar{I}^{\nu}$, по скобка (10), полученная с помощью набора $\left\{I^{1}, \ldots, I^{N}\right\}$, после замены параметров на семействе т-фазньх решений вида $U^{\nu}=U^{\nu}(\overline{\mathbf{U}})$ переходит в скобку, полученную с помощью набора $\left\{\bar{I}^{1}, \ldots, \bar{I}^{N}\right\}$.

Доказательство теорем 1 и 2 полностью совпадает с доказательством тех же теорем для усредненных методом Дубровина-Новикова теоретико-полевых скобок Пуассона (см. [6], [7]) и базируется на ограничении по Дираку скобки Пуассона

$$
\left\{\varphi^{i}(\theta, X), \varphi^{j}\left(\theta^{\prime}, Y\right)\right\}=\sum_{m} B_{(m)}^{i j}(\varphi(X), \varphi(X-\varepsilon), \ldots) \delta(X-Y+m \varepsilon) \delta\left(\theta-\theta^{\prime}\right)
$$

на подмногообразие функций таких, что $\varphi(\theta, X)$ при любом $X$ является функцией из (3) (при этом полагается $\delta(X-Y+m \varepsilon)=\delta(X-Y)+m \varepsilon \delta^{\prime}(X-Y)+\cdots$ при свертке с гладкими функциями). Необходимые условия регулярности этого подмногообразия, а также координаты, в которых производится ограничение, подробно описаны в [6].

В заключение автор благодарит С. П. Новикова и О.И. Мохова за внимание к работе.

\section{СПИСОК ЛИТЕРАТУРЫ}

[1] Уизем Дж. Линейные и нелинейные волны. М.: Мир, 1977. [2] Luke J.C. // Proc. Roy. Soc. London Ser. A. 1966. V. 292. № 1430. P. 403-412. [3] Дубровин Б. А. // Функцион. анализ и его прилож. 1989. Т. 23. № 2. С. 57-59. [4] Дубровин Б. А., Новиков С. П. // Докл. АН СССР. 1983. Т. 270. № 4. С. 781-785. [5] Дубровин Б. А., Новиков С. П. // УМН. 1989. Т. 44. №6. C. 29-98. [6] Maltsev A. Ya.// solv-int/9611008 1996 (to appear). [7] Мальцев А.Я. // УМН. 1997. Т. 52. № 2. С. 177-178.

Институт теоретической физики им. Л. Д. Ландау РАН E-mail: maltsev@itp.ac.ru 